Journal of Computer Science 2 (1): 59-62, 2006

ISSN 1549-3636

(c) 2006 Science Publications

\title{
Location Based Throughput Maximization Routing in Energy Constrained Mobile Ad-hoc Network
}

\author{
${ }^{1} \mathrm{~V}$. Sumathy, ${ }^{2} \mathrm{P}$. Narayanasamy and ${ }^{3} \mathrm{R}$. Prabhu \\ ${ }^{1}$ Government College of Technology, Coimbatore-13, ${ }^{2}$ Anna university, Chennai-25 \\ ${ }^{3}$ Government College of Technology, Coimbatore-13, India
}

\begin{abstract}
In wireless Ad-hoc network, power consumption becomes an important issue due to limited battery power. One of the reasons for energy expenditure in this network is irregularly distributed node pattern, which impose large interference range in certain area. To maximize the lifetime of ad-hoc mobile network, the power consumption rate of each node must be evenly distributed and the over all transmission range of each node must be minimized. Our protocol, Location based throughput maximization routing in energy constrained Ad-hoc network finds routing paths, which maximize the lifetime of individual nodes and minimize the total transmission energy consumption. The life of the entire network is increased and the network throughput is also increased. The reliability of the path is also increased. Location based energy constrained routing finds the distance between the nodes. Based on the distance the transmission power required is calculated and dynamically reduces the total transmission energy.
\end{abstract}

Key words: LBECR, transmission power, reliability, lifetime, throughput

\section{INTRODUCTION}

An Ad-hoc network is a self-organizing wireless network that does not require a pre-established infrastructure. Power consumption in Ad hoc network imposed some limitations in data transmission range. So every node should serve as relays for data transmission. An Ad-hoc network provides robust communication even in hostile environment. This network can be used for military, law enforcement etc...An Ad-hoc network poses a challenge for developing efficient routing protocols. Efficient routing needs complete network topology information. But in Ad-hoc network, topology changes quite often, requiring large exchange of data among the network nodes.

In Ad-Hoc Networks each node must be capable of acting as a router. As a result of the limited radio range of the nodes, a source and destination may have to communicate through a chain of intermediate nodes. Ad-Hoc networks do not have a fixed topology, so route construction and maintenance are continual problems in these networks. The bandwidth is scarce and battery power is limited, it is important to find efficient routing algorithms to create maintain and repair paths with a minimum overhead.

We have proposed a location based throughput maximization routing in energy constrained Ad-hoc networks to reduce the likelihood of data packets being dropped due to less power in the node. Our protocol also increases the lifetime of the networks and nodes.

Existing Proactive routing protocols are not suitable for large size network, because it increases the overhead and it is a table driven method. The advantage is that route information is available whenever needed.

Reactive routing protocols invoke route determination on an on-demand basis. So the routes are not readily available, whenever needed. It increases the delay in large size network to find the routes. So we have considered Hybrid network i.e ZRP. ZRP is suitable for large size network. We have proposed a routing protocol, it finds a reliable and throughput maximization route in ZRP, It increases the network lifetime, decreases the packet drop, increases the packet delivery ratio, extend the lifetime of the node also.

Zone routing protocol: Existing routing protocols based on proactive and reactive methods. Proactive continuously evaluate the routes and the route is already known and can be immediately used. In proactive the traffic is more and converges slowly. The convergence problem is addressed by link state protocol, still the traffic is more.

Hybrid Ad-hoc routing protocols such as ZRP combine both proactive and reactive to achieve a higher level of efficiency and scalability. Proactive method maintains route to destination within a local neighborhood i.e., within the routing zone.

Overview of ZRP: Proactive method maintains the routes by IntraZone routing protocol (IARP) ${ }^{[1]}$. This protocol is used to find the neighborhood node paths, by sending HELLO packets to the nearby nodes.

InterZone routing protocol $(\text { IERP })^{[2]}$ reactively acquires routes for the nodes existing beyond the routing zone. IERP forward queries using the Border cast routing Protocol ${ }^{[3]}(\mathrm{BRP})$. 
In ZRP protocol proactive updates are propagated locally, so the amount of control traffic does not depend on network size. The reactive routing is more efficient and route is discovered on demand.

ZRP can be extended to large size network also. So it is scalable. We have modified ZRP to extend the lifetime of the network and to increase the data delivery ratio.

\section{SYSTEM ENERGY MODEL}

An Ad-hoc network can be modeled by a weighted graph $\mathrm{G}=(\mathrm{N}, \mathrm{L})$, where $\mathrm{N}$ is the set of mobile nodes and $\mathrm{L}$ is the set of full duplex communication links. The weight associated with a link $(u, v) \in \mathrm{L}$ is the energy level of its two endpoints $\mathrm{u}$ and $\mathrm{v}$ and its residual battery energy. $\mathrm{u}$ and $\mathrm{v}$ should be within the transmission range of each other. Suppose a source node $\mathrm{u}$ transmits a packet to a destination node $\mathrm{v}$ and it has a distance $\mathrm{d}_{(\mathrm{u}, \mathrm{v})}$ from $\mathrm{u}$. Then, the minimum transmission energy required at $\mathrm{u}$ is $\mathrm{E}_{(\mathrm{u}, \mathrm{v})}$.

$\mathrm{E}_{(\mathrm{u}, \mathrm{v})}=\mathrm{k} \mathrm{d}^{\alpha}{ }_{(\mathrm{u}, \mathrm{v})}$

Where, $\mathrm{k}$ and $\alpha$ are constants and $\alpha$ varies between 2 and 4. Eq (2.1) represents an approximation model for power consumption at $u$. A residual network $G_{r}=\left(N, L_{r}\right)$ of an Ad-hoc network $\mathrm{G}=(\mathrm{N}, \mathrm{L})$ is defined as follows. The remaining energy of the battery at node $u$ at time $t$ is $\mathrm{E}_{\mathrm{r}}(\mathrm{u})$. The battery capacity at $\mathrm{v}$ initially is $\mathrm{C}(\mathrm{v})$. If a mobile node $v_{j}$ is within the transmission range of $v_{i}$ then $v_{i}$ needs the energy $E\left(v_{i}\right)$ to transmit a packet, then there is a directed edge in $\mathrm{G}_{\mathrm{r}}$ from vi to $\mathrm{vj}$ and the pair of weight assigned to the edge is $\mathrm{kd}^{\alpha}\left({ }_{\mathrm{vi}}, \mathrm{vj}\right),\left(\mathrm{E}\left(\mathrm{v}_{\mathrm{i}}\right)\right.$ $\left.\mathrm{kd}^{\alpha}\left(\mathrm{vi}_{\mathrm{i}} \mathrm{vj}\right)\right), \mathrm{C}\left(\mathrm{v}_{\mathrm{i}}\right)$. Where $\mathrm{d}\left(\mathrm{vi}_{\mathrm{i}} \mathrm{vj}\right)$ is the distance between vi and vj .i.e. $\mathrm{L}_{\mathrm{r}} \subseteq \mathrm{L}$.

Let $\mathrm{P}$ be the residual energy path consist of links $<$ $\mathrm{v}_{0}, \mathrm{v}_{1}>,<\mathrm{v}_{1}, \mathrm{v}_{2},>\ldots,<v_{l-1} . . \mathrm{v}_{l}>$. Each link is provided with the weight $\left(\mathrm{k} \mathrm{d}^{\alpha}{ }_{\mathrm{vi}, \mathrm{vi}+1},\left(\mathrm{Ev}_{\mathrm{i}-\mathrm{k} \mathrm{d}}{ }_{\mathrm{vi}, \mathrm{vi+1}}\right), \mathrm{C}\left(\mathrm{v}_{\mathrm{i}}\right)\right.$ ). . During the Route Discovery, the node is recognized, only when the node energy is greater than or equal to the minimum threshold Energy $\mathrm{E}_{\mathrm{th}}$.

Minimizing the first component of the weighted edge and maximizing the second component of the weighted edge determine the restricted path $\mathrm{P}$.

Location based energy constrained routing: In our routing protocol each node is provided with Global Positioning System. (GPS). The location of the node is obtained from GPS. From the location information each node can calculate the distance to its neighbor node. From the distance the energy required to transmit the data to the neighbors is also calculated by Eq.. (2.1).

Each node maintains the neighbor node list within the zone and its distance in the routing table, by sending the NDP_BEACON packet. When there is a data to transmit, source node will verify the routing table whether the destination is within its zone. If the route to the destination is known, data is send.

Route discovery: When a Source node needs to send a data to a destination but does not have any route information, it searches the route by sending the ROUTE REQUEST (RREQ) packet ${ }^{[4]}$ through peripheral nodes across the network. Each RREQ is send with the distance $\mathrm{kd}^{\alpha}\left({ }_{\mathrm{vi}}\right.$, vj $)$ from source to the intermediate node and the source nodes remaining energy $\left(\mathrm{kd}^{\alpha}{ }_{\mathrm{vi}, \mathrm{vi}+1}, \mathrm{Ev}_{\mathrm{i}-} \mathrm{kd}^{\alpha}{ }_{\mathrm{vi}, \mathrm{vi}+1} / \mathrm{c}\left(\mathrm{v}_{\mathrm{i}}\right)\right)$. where $\mathrm{i}$ and $\mathrm{j}$ are neighbor nodes.

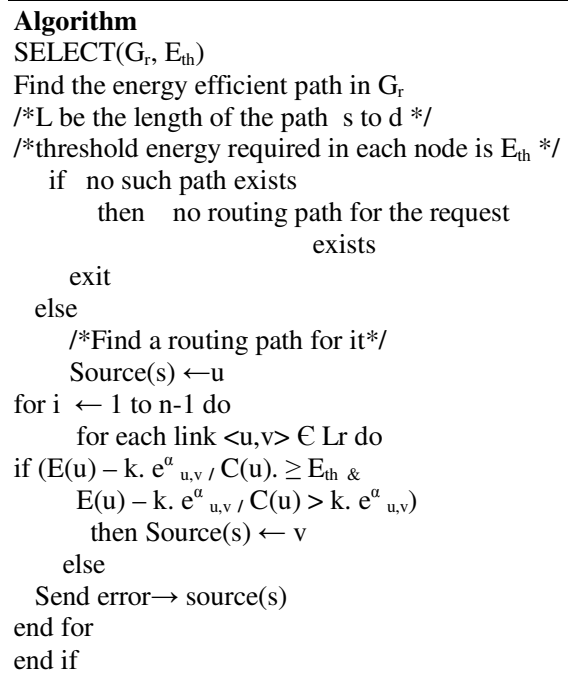

Intermediate nodes receiving the RREQ packet update the information for the source node and set up backwards pointers to the source node in their route tables and forwarded. The packet from the destination to the source follow a path, which was set up by the route request packet .The RREQ also contains source node's IP address, current sequence number and broadcast ID. The source nodes sequence number is used to maintain freshness information about the reverse route to the source.

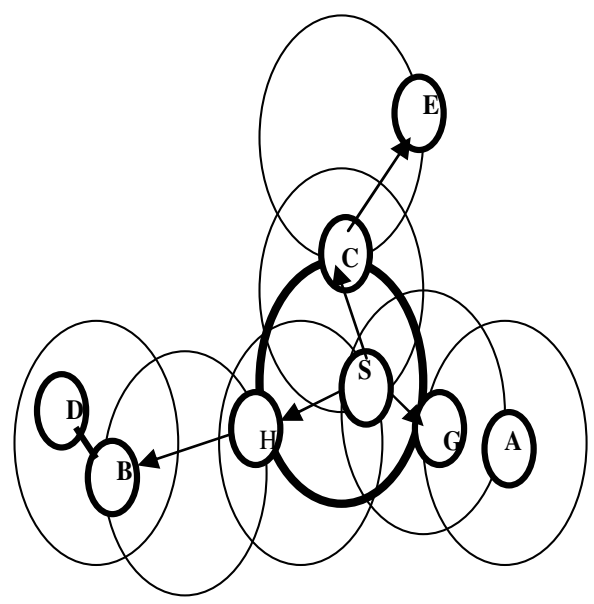

Fig. 1: Route discovery 
For example if source node $\mathrm{S}$ has data for D. S first checks whether D is within its routing zone. If so, $\mathrm{S}$ already knows the route to node $\mathrm{D}$. Otherwise $\mathrm{S}$ sends a query (RREQ) to all its peripheral nodes.(C,G,H) Now the node send the query with the distance $\mathrm{kd}^{\alpha}{ }_{(\mathrm{vi}, \mathrm{vj})}$ and the nodes remaining battery energy to its peripheral nodes i.e. $\left(\mathrm{kd}^{\alpha}{ }_{\mathrm{vi}, \mathrm{vi}+1}, \mathrm{Ev}_{\mathrm{i}}-\mathrm{kd}^{\alpha}{ }_{\mathrm{vi}, \mathrm{vi}+1} / \mathrm{c}\left(\mathrm{v}_{\mathrm{i}}\right)\right)$

Each node reads the remaining battery energy of the previous node from RREQ packet and compare with its remaining energy during the route discovery. Only the minimum battery energy is retained and transmitted to the neighbor node. For example in Fig. 1 if remaining battery energy of node $\mathrm{H}$ is greater than $\mathrm{S}$ then $\mathrm{S}$ nodes energy is retained and included in the route RREQ query packet and forwarded to the neighbor. This is to avoid the link break due to less energy in the node. The logic behind this is, as soon as a single link in a path is disconnected, the entire path is invalidated. Multiple paths reach the destination D then it sends the reply by backward path.

Route reply: The RREP route reply packet is send by the destination to the source. It unicasts a RREP packet back to the source through the backward pointer. Multiple RREP ${ }^{[5]}$ reaches the source. The source selects a path with maximum remaining battery capacity among the multiple paths with minimum remaining battery capacity of the path. It increases the network lifetime, reliability and increases the packet delivery ratio also.

Route maintenance: The intermediate node in the path monitors the decrease in its remaining battery energy, from the time of route discovery as a result of forwarding packets along this route.

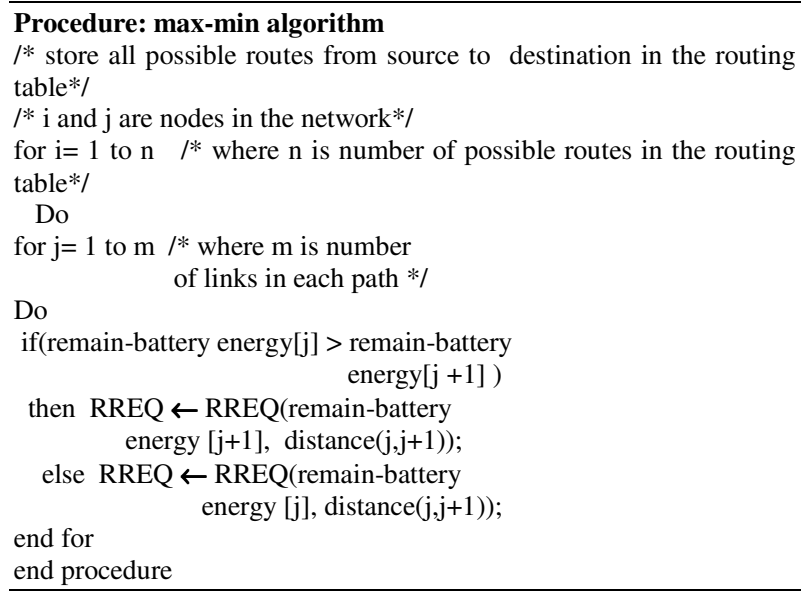

When the node energy decreases below a threshold or if a node moves out of the transmission range of its neighbor node, the intermediate node cannot forward the data packet. The node sends a route error ROUTE_ERR packet back to the source. This route error message forces the source to initiate route discovery again. The route discovery is dependent on the remaining battery capacity of the current node. So this is a local approach for route maintenance.

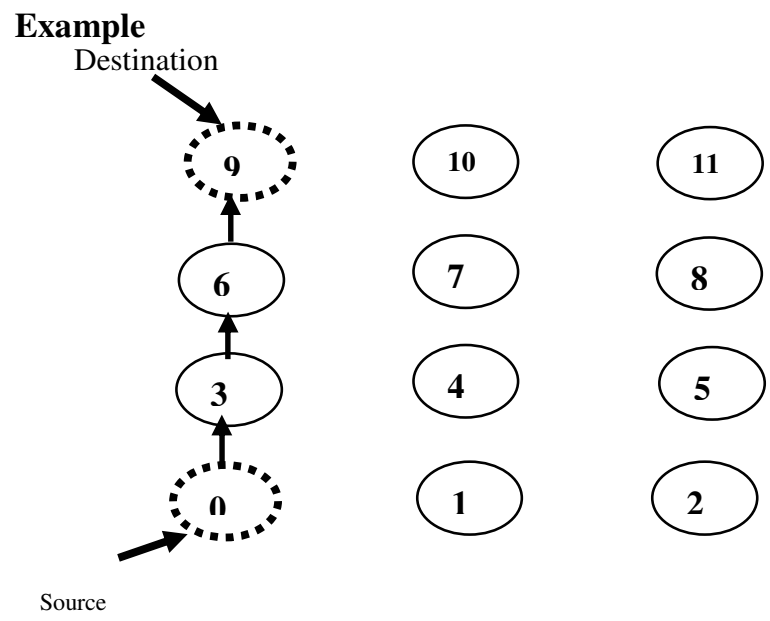

Fig. 2: CBR-traffic from source to destination

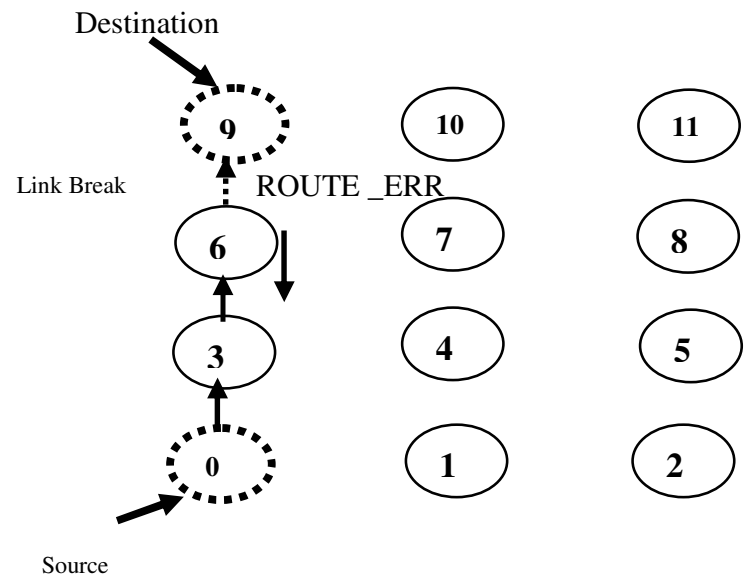

Fig. 3: CBR-traffic from source to destination with link break

In the example shown in Fig. 2 the path is (0-3-6-9). Node 0 is source and node 9 is destination. Node 6 cannot send the packet to 9 if the remaining battery energy in node 6 is less than the threshold $E_{\text {th }}$. Now node 6 will initiate the ROUTE_ERR packet and shown in Fig. 3.

Table 1: Fixed simulation parameters

\begin{tabular}{ll}
\hline Node $(\mathrm{N})$ & $9,12,22$ \\
Network Coverage Area & $700 X 700 \mathrm{~m}$ \\
Node Placement & Random \\
Mobility & Random Way point \\
Transmission Radius & $250(\mathrm{~m})$ \\
Data Type & $\mathrm{CBR}$ \\
Simulation Time & $20,40 \mathrm{~s}$ \\
Data Transmission Rate & $2 \mathrm{Mbs}$ \\
Packet Size & 512 bytes \\
\hline
\end{tabular}

Simulation results and comparison: In our routing protocol we have analyzed the network throughput and 
the network node lifetime. Compared to existing ZRP our Location based energy constrained routing protocol performs better, decreases the packet drop ratio.

\begin{tabular}{lllll} 
Table 2: & \multicolumn{3}{c}{ Node Life time comparison with simulation time $20 \mathrm{~s}$} \\
\hline Topology & $\begin{array}{l}\text { Node lifetime } \\
\text { in LBZRP(s) }\end{array}$ & $\%$ & $\begin{array}{l}\text { Node lifetime } \\
\text { in ZRP(s) }\end{array}$ & $\%$ \\
\hline 9 & 18.5 & 92.5 & 11.8 & 59 \\
12 & 20 & 100 & 13 & 65 \\
22 & 20 & 100 & 16 & 80 \\
\hline
\end{tabular}

Table 3: Throughput comparison with threshold 0.2 and simulation time $20 \mathrm{~s}$

\begin{tabular}{lll}
\hline Topology & $\begin{array}{l}\text { LBZRP packet } \\
\text { Delivery ratio \% }\end{array}$ & $\begin{array}{l}\text { ZRP Packet } \\
\text { Delivery ratio \% }\end{array}$ \\
\hline 9 & 98.87 & 61.29 \\
12 & 98.5 & 59.09 \\
22 & 97.7 & 58.5 \\
\hline
\end{tabular}

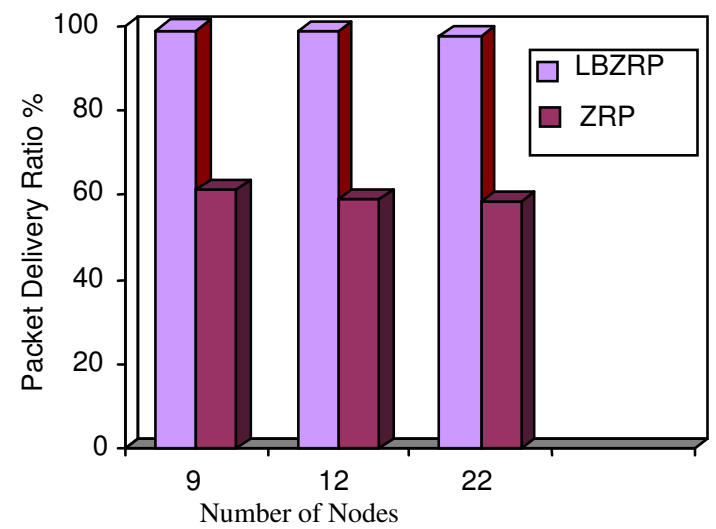

Fig. 4: Throughput comparison for the simulation time $20 \mathrm{~s}$ with threshold 0.2

In our location based protocol the lifetime of the network node is increased by $30 \%$ compared to ZRP. The Throughput is also increased by $39 \%$ in our location based throughput maximization routing shown in Fig. 4 and 5.

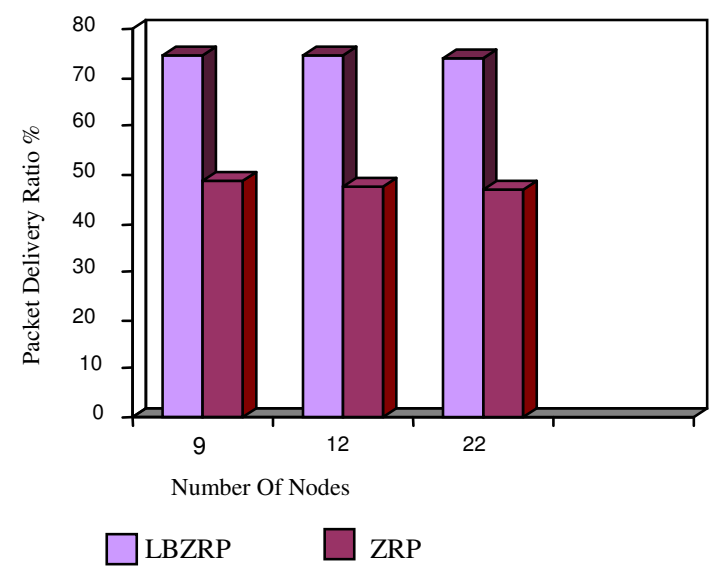

Fig. 5: Throughput Comparison for the Simulation Time 20s with threshold energy $6 \mathrm{~J}$

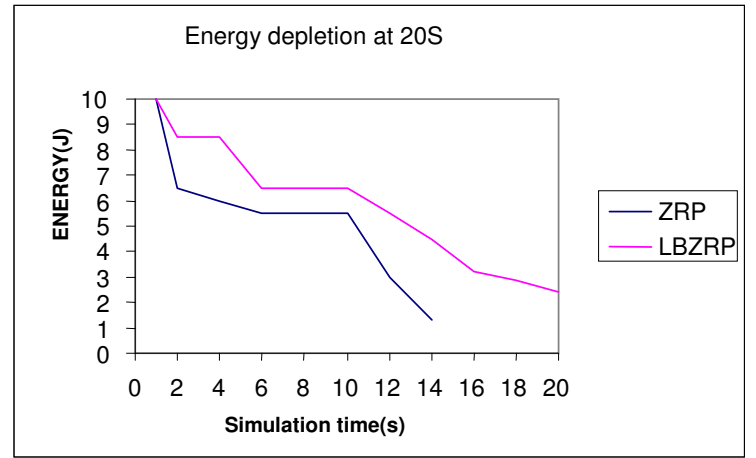

Fig. 6: Energy depletion in network

Simulation parameters are shown in Table 1-3.The Network lifetime is increased by $48 \%$ in our location based energy constrained routing. The node with maximum remaining energy is considered for path selection. So the remaining energy in the node is also maximum, it increases the node lifetime, network lifetime and shown in Fig. 6.

\section{CONCLUSION}

In our Location based energy constrained routing protocol compared to original ZRP, the network life time is increased by $48 \%$,each node energy is also saved by $30 \%$. The packet delivery ratio also increased by $39 \%$.

Future work: The stale routes in the routing table can be removed dynamically. In our protocol all the nodes are considered as Homogeneous nodes. We can consider heterogeneous nodes and the network can be analyzed.

\section{REFERENCES}

1. Haas Zygmunt, J., R. Pearlman Marc and P. Samar, 2001. IntrAzone Routing Protocol (IARP). IETF Internet Draft, draft-ietf-manet-iarp-01.txt.

2. Haas Zygmunt, J., R. Pearlman Marc and P. Samar, 2001. IntErzone Routing Protocol (IERP). IETF Internet Draft, draft-ietf-manet-ierp-01.txt.

3. Haas Zygmunt, J., R. Pearlman Marc and P. Samar, 2001. The Bordercast Resolution Protocol (BRP). IETF Internet Draft, draft-ietf-manet-brp-01.txt.

4. Lan Wang, Stephen Olariu, 2003. A two-zone hybrid routing protocol for mobile ad-hoc networks.

5. Nicklas Beiger, 2002. Zone Routing Protocol (ZRP). Helsinki University of Technology, Finland. 